\title{
Design and Characterization of a CMOS 3-D Image Sensor Based on Single Photon Avalanche Diodes
}

\author{
Cristiano Niclass, Student Member, IEEE, Alexis Rochas, Pierre-André Besse, and Edoardo Charbon, Member, IEEE
}

\begin{abstract}
The design and characterization of an imaging system is presented for depth information capture of arbitrary three-dimensional (3-D) objects. The core of the system is an array of $32 \times 32$ rangefinding pixels that independently measure the time-of-flight of a ray of light as it is reflected back from the objects in a scene. A single cone of pulsed laser light illuminates the scene, thus no complex mechanical scanning or expensive optical equipment are needed. Millimetric depth accuracies can be reached thanks to the rangefinder's optical detectors that enable picosecond time discrimination. The detectors, based on a single photon avalanche diode operating in Geiger mode, utilize avalanche multiplication to enhance light detection. On-pixel high-speed electrical amplification can therefore be eliminated, thus greatly simplifying the array and potentially reducing its power dissipation. Optical power requirements on the light source can also be significantly relaxed, due to the array's sensitivity to single photon events. A number of standard performance measurements, conducted on the imager, are discussed in the paper. The 3-D imaging system was also tested on real 3-D subjects, including human facial models, demonstrating the suitability of the approach.
\end{abstract}

Index Terms-CMOS avalanche photodiodes, highly sensitive photodetectors, LIDAR, photon timing, rangefinder, single photon detectors, SPAD arrays, time-correlated measurements, 3-D image sensor, 3-D vision.

\section{INTRODUCTION}

$\mathbf{V}$ ISION systems for capturing and rendering three-dimensional (3-D) images have existed since the dawn of photography. Despite considerable technological progress, especially in rendering techniques, 3-D vision has been relegated to research, particularly in robotics, and in relatively low-volume applications. Recently, the appearance of low-cost digital still and video cameras has reversed this trend and has accelerated the push to create low-cost and compact 3-D native vision systems. Currently, a number of novel and potentially high volume applications requiring fast and precise depth map evaluation are being devised and the market share of software for capturing, processing, and rendering 3-D images has grown exponentially. More recent applications include face recognition, virtual keyboards, object and person monitoring, land and sea surveying, virtual reality games, nonionizing medical tomographic imagers, stage and choreography analysis tools, etc. Cost concerns have prompted the emergence of a new

Manuscript received December 8, 2004; revised February 23, 2005. This work was supported by the Swiss National Science Foundation under Grant 620-066110.

The authors are with the School of Computer and Communication Sciences and the School of Engineering, Ecole Polytechnique Fédérale de Lausanne (EPFL), 1015 Lausanne, Switzerland (e-mail: edoardo.charbon@epfl.ch).

Digital Object Identifier 10.1109/JSSC.2005.848173 generation of solid-state imagers that can achieve the desired accuracy and speed in compact implementations.

Non-stereoscopic 3-D vision techniques can be divided into three main classes: triangulation, interferometry, and time-offlight (TOF). Systems based on triangulation evaluate depth by illuminating a tiny spot of the scene with a laser ray. The distance of that spot to the sensor is inferred from the known angle of incidence of the reflected light [1]. The main disadvantages of such systems are the speed requirement on the sensor (usually a conventional CMOS or CCD camera), power dissipation, and the need for highly tuned precision moving parts. Alternatively, interferometric methods may be used in 3-D vision. However, to date, the axial range is still limited and the cost of such systems is still prohibitive [2].

TOF methods are available today in two main variants: modulation- or pulsed-based methods. Modulation-based TOF range finders measure the phase difference between a modulated laser source and the reflected wave. In these imagers, megahertz modulation frequencies, in conjunction with homodyne phase discrimination and averaging at the pixel level, have been successfully used to relax circuit specifications [3], [4]. One of the problems of this method is the requirement for a relatively powerful laser or LED source, while accuracy is severely limited by the speed at which the sensor can be clocked. In pulsed-type TOF methods, the round-trip time of a single burst of light is measured. The main advantage of these methods over modulated-type TOF is that a range of operation of a few meters to several kilometers can be achieved avoiding the use of different modulation frequencies.

To achieve millimetric accuracies, pulsed-type TOF rangefinders must have picosecond time discrimination capability, high detection speed or, conversely, low jitter, and low ray divergence. To date, conventional CMOS detectors can meet these requirements only at a cost of highly sophisticated detection schemes and very powerful laser sources [5]. Complex non-CMOS technologies are a good alternative but they generally prevent integration of accurate time discriminators on chip, unless hybrid, potentially expensive solutions are adopted [6]. Another alternative is the use of micro-optical scanning systems together with a single highly sensitive photon counting device [7]. This solution however requires laboratory-style optical set-ups.

In [9], an $8 \times 4$ array of single photon avalanche diodes (SPADs) integrated in a conventional CMOS technology was presented. Its potential for 3-D imaging was demonstrated in [10]. However, this solid-state sensor still suffered from a limited lateral resolution due to the reduced number of pixels. In this paper we report on a solid-state 3-D imager based on 
the pulsed-type TOF. The sensor is implemented in CMOS technology and it consists of an array of SPADs, capable of performing 1024 independent distance measurements. An external low-cost CMOS time discriminator based on a time-to-digital converter is employed to achieve the proper accuracies. A uniform illumination of the scene is accomplished using a single intentionally uncollimated laser beam. This method enabled us to achieve a very compact imaging solution that requires no mechanical micro-optical scanning device. The SPAD's high sensitivity enables the sensor to detect low levels of reflected light. Therefore, inexpensive laser sources with milliwatt peak power can be used for ranges up to several meters.

The jitter properties of SPADs allow a time discriminator to meaningfully measure picosecond time intervals. Depth and intensity can be evaluated by the same pixel, thus the imager generates 3-D images with little or no alignment error between the two measurements. Tight speed and accuracy specifications do not impact system complexity as in other approaches, while keeping power dissipation to a minimum thanks to the inherent simplicity of the sensor design. Not less importantly, due to the reduced power of the laser source, it is possible to guarantee strict eye safety operation of the system.

This paper is organized as follows. Section II describes the principle of operation of the optical detectors of the sensor. Sections III and IV present the sensor architecture and rangefinder set-up. Section V outlines the imager performance.

\section{Single Photon Avalanche Diodes (SPADs)}

\section{A. Principle of Operation and CMOS Implementation}

A p-n junction reversed biased above its breakdown voltage $\left(V_{b d}\right)$ allows single photon detection [8]. Reverse biasing a $\mathrm{p}$-n junction beyond its breakdown voltage should intrigue those who are familiar with the current-voltage characteristic of a typical p-n junction. The explanation requires a study of temporal evolution of the phenomenon. Let us consider a $\mathrm{p}$-n junction in complete darkness. If one suddenly applies a reversed bias voltage above $V_{b d}$, there will be no breakdown current until a carrier enters the depletion region. During this short period of time, a very high electric field exists within the junction forming the avalanche's multiplication region. Suppose now that a photon is absorbed at this moment in the diode's depletion region. The primary carriers resulting from the absorption of the photon may generate an extremely high number of secondary electron-hole pairs by impact ionization, thus causing the diode's depletion capacitance to be rapidly discharged. As a result a sharp current pulse is generated and can be easily measured. This mode of operation is commonly known as Geiger mode.

Unfortunately, typical p-n junctions, as those used in conventional CMOS or CCD imagers, are not compatible with this mode of operation since they do not allow high voltage biasing without producing an instantaneous breakdown current. The reason for this behavior is that the peak electric field is located only in the diode's periphery, thus causing premature breakdown [8].

Biased just below $V_{b d}$, an avalanche photodiode is physically a specific design of a $\mathrm{p}-\mathrm{n}$ junction so that premature breakdown

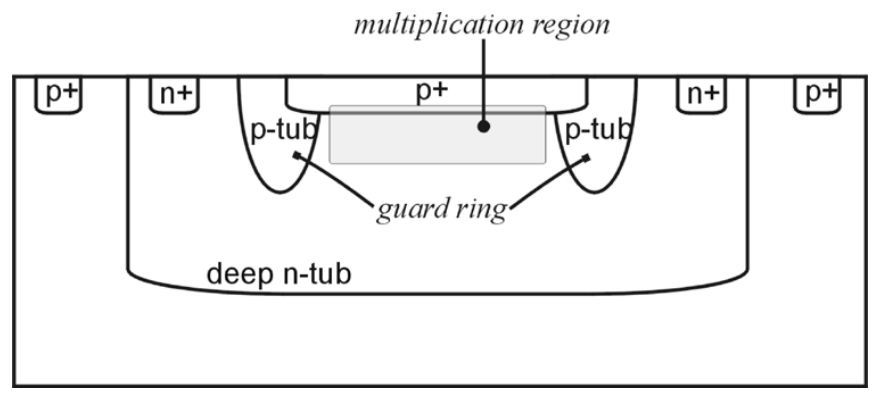

Fig. 1. Single photon avalanche diode (SPAD) cross-section.

is avoided and a planar multiplication region is formed within the junction. SPADs, on the other hand, are such a p-n junction biased far above $V_{b d}$ and operating in the so-called Geiger mode.

The designed SPAD, shown in Fig. 1, was fabricated using a high-voltage $0.8 \mu \mathrm{m}$ CMOS process. It is a dual $\mathrm{p}+/$ deep n-tub/p-substrate junction. The upper $\mathrm{p}+/$ deep n-tub junction provides the multiplication region where the Geiger breakdown occurs. The fabrication process is a $2 \mathrm{M} / 2 \mathrm{P}$ twin-tub technology on a p-substrate allowing an operating voltage of 2.5 to $50 \mathrm{~V}$. Premature breakdown is avoided by surrounding the photodiode with a guard ring of relatively lightly doped diffusion. The purpose is to eliminate abrupt doping profiles and corner effects, thereby reducing electric potential gradients. A useful feature of this technology is the availability of a p-tub implantation to create such a ring surrounding the $\mathrm{p}+$ region anode [11]. The breakdown voltage $V_{b d}$ of the p+/deep n-tub junction is typically $25.5 \mathrm{~V}$. A larger bias voltage $V_{b d}+V_{e}$ must be applied on the diode to operate in single photon detection mode. $V_{e}$ is known as excess bias voltage.

\section{B. SPAD Performance Parameters}

Avalanche photodiodes, which are biased just below $V_{b d}$, operate in the linear mode and have a finite multiplication gain. They suffer from excess noise generated by statistical variation of the multiplication gain. SPADs, on the other hand, are not concerned with these gain fluctuations since only photons that give rise to a very high number of carriers are counted as a logic signal. Nevertheless, the statistical variation of the avalanche buildup is translated onto a detection probability. Indeed, the probability of detecting a photon hitting the SPAD's surface, known as the Photon Detection Probability (PDP), depends on the diode's quantum efficiency and the probability for an electron or for a hole to trigger an avalanche. Usual values for PDP in CMOS SPADs are in the range of $1 \%$ to $30 \%$ [8].

Unlike in linear mode photodiodes, in SPADs, the signal amplitude does not provide intensity information since a current pulse has the same amplitude whether it had been triggered by a single or multiple photons. Intensity information is obtained by counting the pulses during a certain period of time or by measuring the mean time interval between successive pulses. The same mechanism may be used to evaluate noise. Thermally or tunneling generated carriers within the p-n junction, which generate dark current in linear mode, can trigger avalanche pulses. In Geiger mode, they are indistinguishable from regular photon-triggered pulses and they produce spurious pulses at a frequency known as Dark Count Rate (DCR). As dark current 
in linear mode, DCR strongly depends on temperature. DCR is an important parameter for imagers since it defines the minimal detectable signal, thus limiting the dynamic range of the imager.

Another source of spurious counts is represented by afterpulses, which can be understood as secondary false counts. They are due to carriers temporarily trapped near the multiplication region that are released after an arbitrary time interval, thus re-triggering a Geiger event. After-pulses depend on the trap concentration as well as on the number of carriers generated during a Geiger pulse. The number of carriers depends, in turn, on the diode's parasitic capacitance and on the external circuit, which is usually the circuit used to quench the avalanche. Typically, the quenching process is achieved by temporarily lowering the bias voltage below $V_{b d}$. In order to optimize after-pulse probability, the number of carriers generated per avalanche need to be minimized. Acceptable numbers are in the range of hundreds of thousands of electron-hole pairs. Once the avalanche has been quenched, the SPAD needs to be biased again above $V_{b d}$ so that it can detect subsequent photons. The time required to quench the avalanche and recharge the diode up to $90 \%$ of its nominal excess bias is defined as the dead time. This parameter should be as low as possible as it restricts the maximal rate of detected photons, thus limiting the dynamic range of the image sensor.

Another important property of SPADs is the ability of accurately detecting the arrival time of photons. The statistical fluctuation of the time interval between the arrival of photon at the sensor and the output pulse leading edge is defined as the timing jitter or timing resolution. In this definition, the term resolution stands for accuracy as it is often suggested in the literature [7], [8]. In a SPAD, the timing jitter mainly depends on the time a photogenerated carrier requires to be swept out of the absorption zone into the multiplication region. This parameter is of great importance for TOF-based 3-D vision.

During an avalanche, some photons can be emitted due to the electroluminescence effect [12]. These photons may be detected by neighboring pixels in an array of SPADs. The probability of this effect is defined as the optical crosstalk probability. Such probability is significantly attenuated in fully integrated arrays of SPADs, if compared to hybrid versions. This is due to the fact that the diode's parasitic capacitance in the integrated version is orders of magnitude smaller than the hybrid solutions, thus reducing the energy peak dissipated during a Geiger event. Electrical crosstalk on the other hand is produced by the fact that photons absorbed beyond the p-n junction, deep in the substrate, generate carriers which can diffuse to neighboring pixels. The probability of occurrence of this effect, whether it is optical or electrical, defines the crosstalk probability.

\section{IMAGE SENSOR}

\section{A. The Digital Pixel}

The pixel consists of a circular SPAD and a $5 \mathrm{~T}$ configuration as shown in Fig. 2. The SPAD, which is based on [8], operates in passive quenching. The $\mathrm{p}+$ anode is biased at a high negative voltage $V_{p}$ equal to $-25.5 \mathrm{~V}$. This voltage is common to all the pixels in the array. The deep n-tub cathode is connected to the

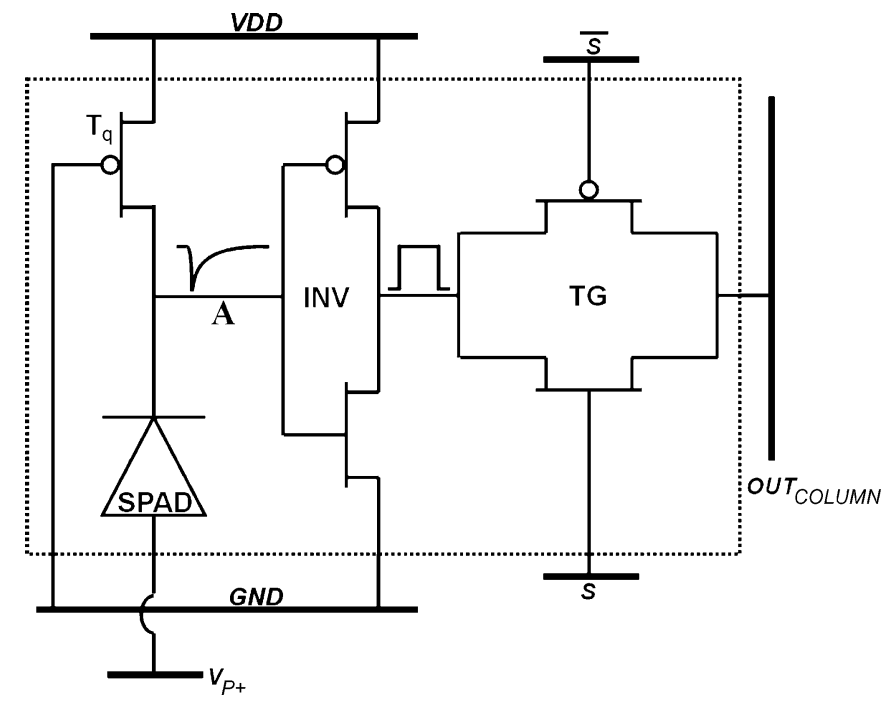

Fig. 2. Schematic of $5 \mathrm{~T}$ digital pixel.

power supply $V_{\mathrm{DD}}=5 \mathrm{~V}$ through a long channel p-mos transistor $\mathrm{T}_{q}$, which eliminates the need for a quenching resistance as proposed in [8]. The excess bias voltage $V_{e}$ is thus equal to $\left|V_{p+}\right|+V_{\mathrm{DD}}-V_{b d}=5 \mathrm{~V}$.

Upon photon arrival, the breakdown current discharges the depletion region capacitance, reducing the voltage across the SPAD to its breakdown voltage. The $\mathrm{W} / \mathrm{L}$ ratio of $\mathrm{T}_{q}$ is set to provide a sufficiently resistive path to quench the avalanche process. In our design, the channel resistance is larger than $200 \mathrm{k} \Omega$ for drain-source voltage $V_{d s}$ between $G N D$ and $V_{\mathrm{DD}}$. After avalanche quenching, the SPAD recharges through $\mathrm{T}_{q}$ and progressively recovers its photon detection capability.

The time required for quenching the avalanche and restoring the operating bias, i.e., the dead time, is typically less than $40 \mathrm{~ns}$ for the $5 \mathrm{~T}$ digital pixel. At node $\mathrm{A}$, an analog voltage pulse of amplitude $V_{e}$ reflects the detection of a single photon. The inverter stage $I N V$ converts this analog voltage pulse into a digital pulse. The transistor aspect ratios of $I N V$ are designed to set the input threshold voltage at $3.5 \mathrm{~V}$. The transmission gate $T G$ feeds the detection signal to the column output line when $\mathrm{s}=V_{\mathrm{DD}}$ and $\bar{s}=G N D$ (read phase). The pixel outputs a digital signal reflecting a photon arrival with picosecond precision. The near-infinite internal gain inherent to Geiger mode operation leads to no further amplification and the pixel output can be routed directly outside the chip. In this paper, to the best of our knowledge, we are reporting the first scalable and intrinsically digital pixels implemented in CMOS process for imaging applications. Unlike other previously published digital pixels [13], [14], this configuration includes no analog components and no local or global A/D converters. Fig. 3 shows the layout of a pixel consisting of the photodiode, the quenching circuitry and the column access. The $5 \mathrm{~T}$ digital pixel occupies a square area of $58 \mu \mathrm{m} \times 58 \mu \mathrm{m}$. The active area of the SPAD is $38 \mu \mathrm{m}^{2}$.

\section{B. Sensor Architecture}

The functional diagram of the sensor is shown in Fig. 4. The sensor array consists of $32 \times 32$ pixels and requires two power supply buses $V_{\mathrm{DD}}=5 \mathrm{~V}$ and $\mathrm{V}_{\mathrm{p}+}=-25.5 \mathrm{~V}$. Digital pixels 


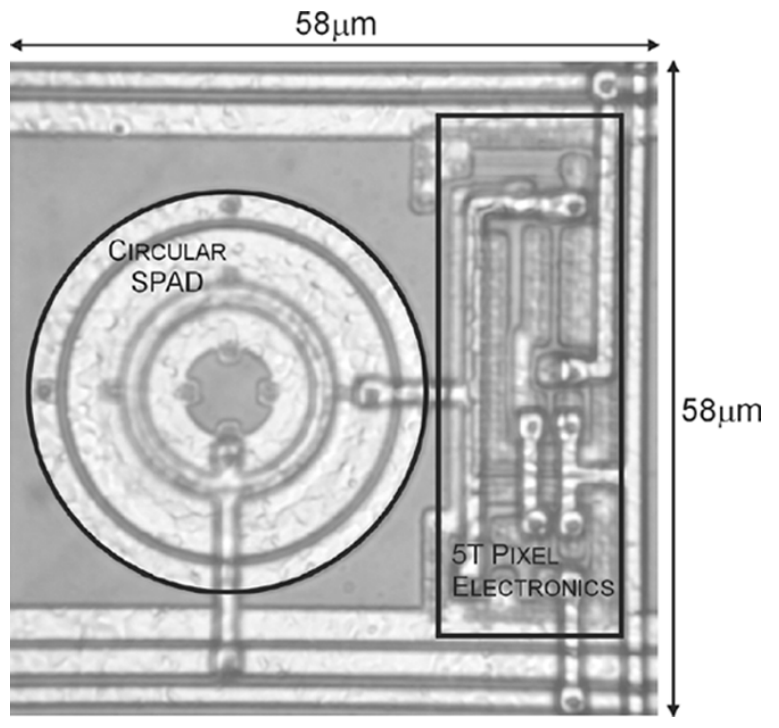

Fig. 3. Microphotograph of SPAD pixel.

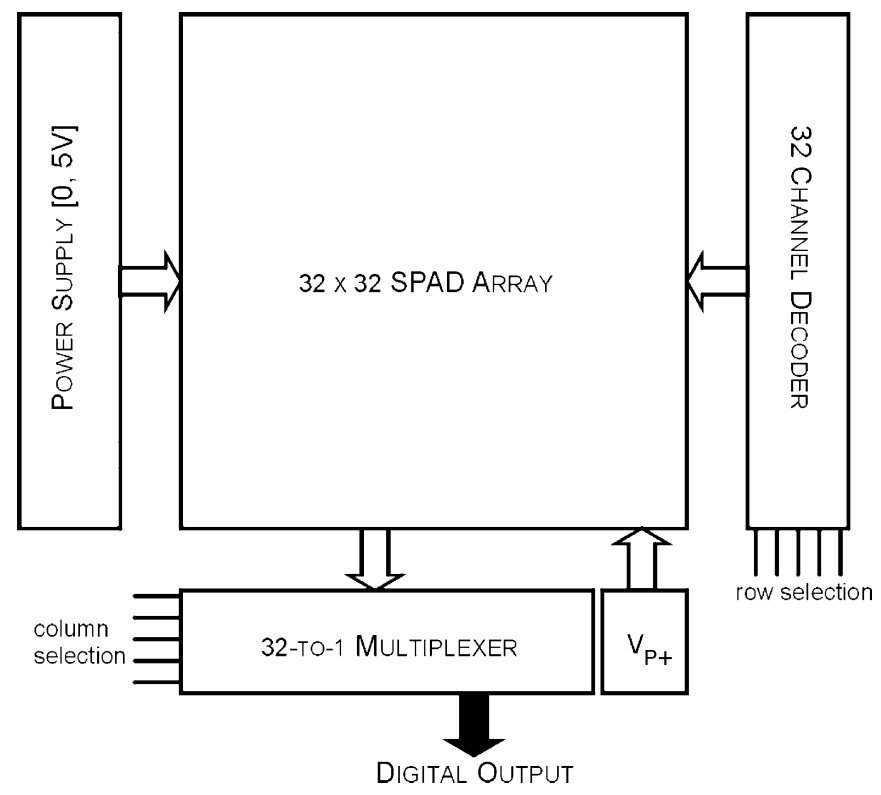

Fig. 4. Functional diagram of fabricated chip.

allow the readout circuitry to be relaxed with respect to noise considerations. Therefore, no amplification, no Sample\&Hold, and no A/D converter are necessary. In this implementation, the readout circuitry consists of a 32-channel decoder for row selection and a 32-to-1 multiplexer for column selection.

A fast 15 bit linear feedback shift register counter has been implemented on-chip and can be connected to the readout circuitry for the imager to operate in intensity or 2-D mode. A microphotograph of the chip is shown in Fig. 5. The chip size is $7 \mathrm{~mm}^{2}$.

\section{RANGEFINDING METHOD}

\section{A. Measurement Setup}

The rangefinding method used in this sensor is based on pulsed-type TOF. Depth is computed as $d=\operatorname{TOF}(c / 2)$, where $c$ is the speed of light. The system has been designed so that

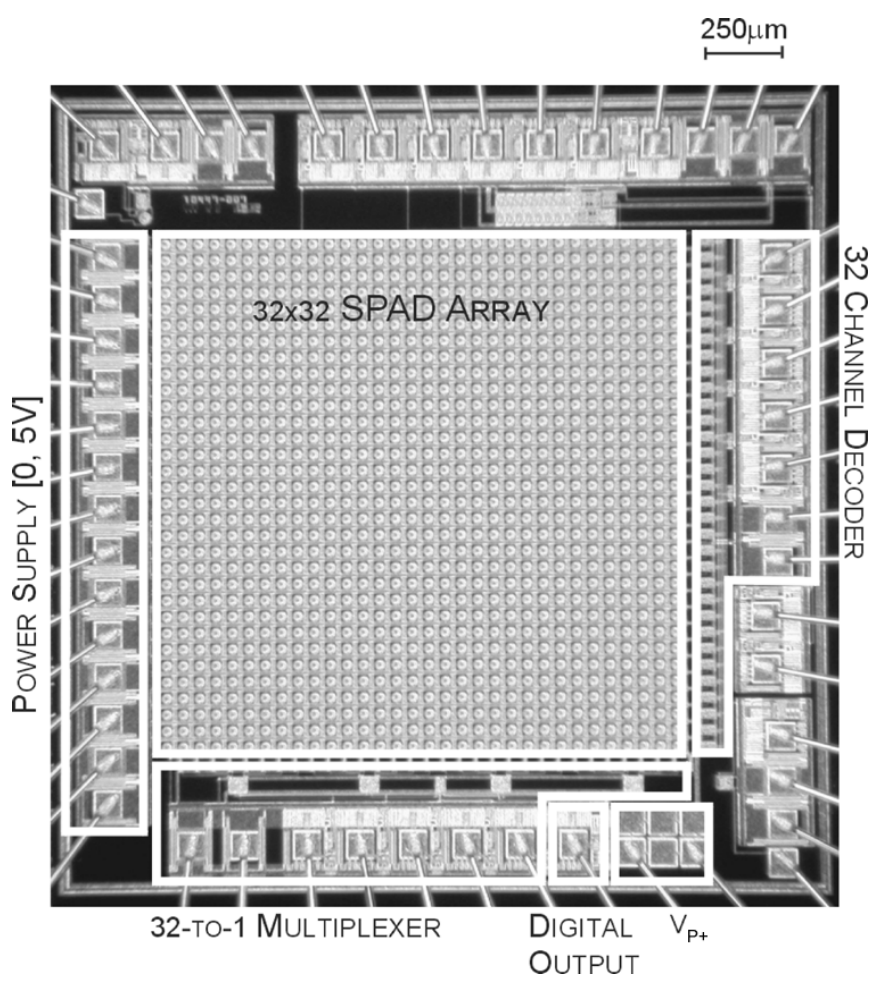

Fig. 5. Microphotograph of the image sensor.

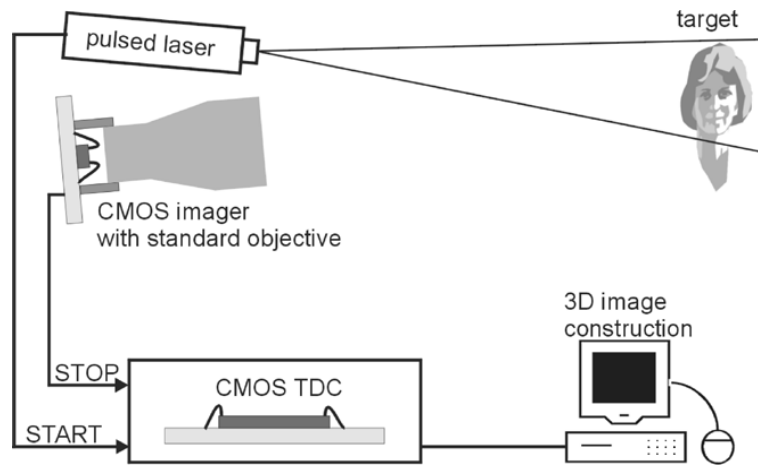

Fig. 6. 3D camera set-up (not to scale).

distance measurements can be obtained keeping the overall sensor complexity to a minimum.

The electro-optical setup is described in Fig. 6. The CMOS imager sensor was mounted on a printed circuit board and coupled with a standard camera objective. The scene was illuminated by a uniform cone of light created using a single intentionally uncollimated laser beam reaching every point of the surface. The laser source was a $635 \mathrm{~nm}$ pulsed diode laser whose repetition rate $f_{R}$ was $50 \mathrm{MHz}$, pulsewidth $T_{P}$ of $150 \mathrm{ps}$, and peak power $P_{S}$ of $100 \mathrm{~mW}$. A time-to-digital converter (TDC), TDC-F1 model from ACAM, was used for time interval measurement. The START signal was given by the synchronization output of the laser whereas the STOP signal was connected to the digital output of the $32 \times 32$ image sensor. The TDC, also implemented in CMOS technology, exhibited a timing resolution of $120 \mathrm{ps}$ and a $1-\sigma$ timing uncertainty of 110 ps. Pixel addressing was performed by an external acquisition card. 
Ref. [7] presents a TOF-based scanner rangefinder using a SPAD. Depth measurements for each point in the scene were performed in sequence using a scanner apparatus. In addition, in order to avoid skews in the distance measurement, TOF was computed with respect to an internal optical reference, i.e., a mirror. The reference mirror shared the same optical path as the target through a beam splitter. In this paper, depth measurements are independently performed for each point in the scene by means of the SPAD array, though serially, due to the implemented readout circuitry. The optical reference path is replaced by a range image of a background plane of $2 \times 2 \mathrm{~m}^{2}$ which is captured using the same setup. The reference image is electronically stored and used in the computation of subsequent images, thus eliminating the pixel-to-pixel skews.

\section{B. Measurement Data Processing}

Although CMOS SPADs exhibit excellent timing jitter properties, typically a few tens of picoseconds, this accuracy is insufficient to reach millimetric precision. Depth accuracy is computed as $\sigma(d)=c \sigma(\tau) / 2$, where $\sigma(\tau)$ is the uncertainty of a single time measurement $\tau$. Assuming that $M$ TOF measurements $\left\{\tau_{j}\right\}, j=1, \ldots, M$ can be approximated as a collection of independent and identically distributed random variables, then, using simple averaging, the system time uncertainty is $M^{-1 / 2} \sigma(\tau)$, thus the distance accuracy can be theoretically increased by the square root of the number of measurements.

In addition, in order to reduce the influence of DCR and background illumination on the depth measurement, a simple processing algorithm was implemented. Each depth measurement was computed building a histogram using a set of $M$ TOF measurements. Since DCR and background illumination pulses are not correlated to the laser source, these pulses produce a noise level which is spread out over the entire histogram range. The signal on the other hand generates a peak in the histogram whose width is $\sigma(\tau)$ and can be easily isolated from noise by applying a threshold operation (see Fig. 10). Finally, depth is obtained by determining the average TOF value of the signal measurements. Note that histogram generation and computation are easily implemented either in software or hardware and they are well-suited for real-time processing.

\section{Measurement Results}

\section{A. Image Sensor}

The performance parameters of a SPAD-based image sensor differ from the conventional approach which is typically limited by the analog readout circuitry noise and the mismatch parameters in the amplification and conversion stages.

Fig. 7 shows the measured PDP as a function of the photon wavelength for a typical pixel with a nominal $V_{e}$ of $5 \mathrm{~V}$ at room temperature. It is larger than $20 \%$ between $430 \mathrm{~nm}$ and $570 \mathrm{~nm}$ with a peak at $26 \%$ at $460 \mathrm{~nm}$. At $700 \mathrm{~nm}$, the PDP is still $8 \%$ without any post process treatment of the silicon chip surface. In Fig. 8, a typical signal measurement across the sensor array is illustrated. The sensor was illuminated with a uniform light of intensity $2.5 \times 10^{-5} \mathrm{~W} / \mathrm{cm}^{2}$ to validate the PDP uniformity. All pixels behaved identically in terms of PDP and dead time.

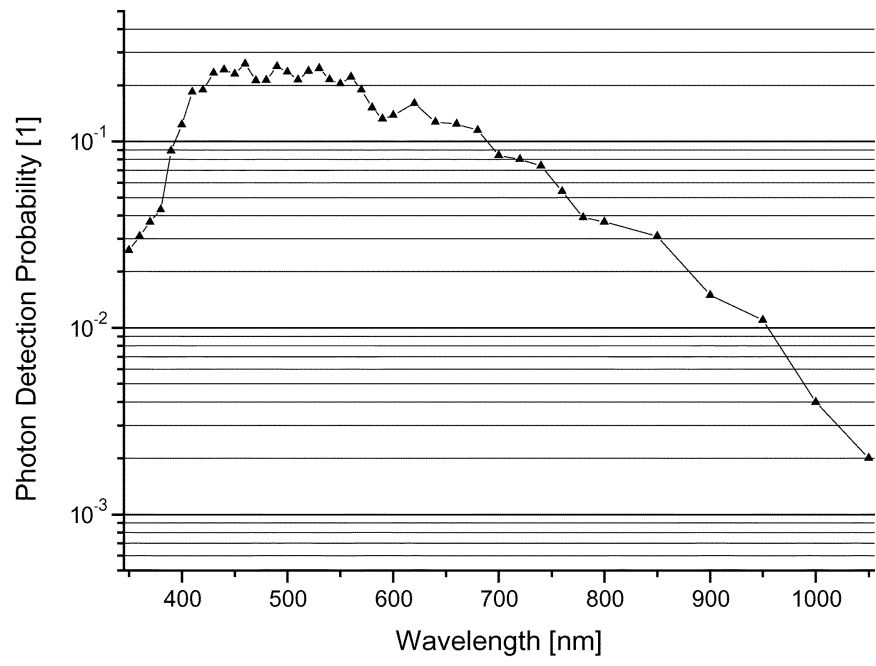

Fig. 7. Photon detection probability of a typical pixel for nominal excess voltage.

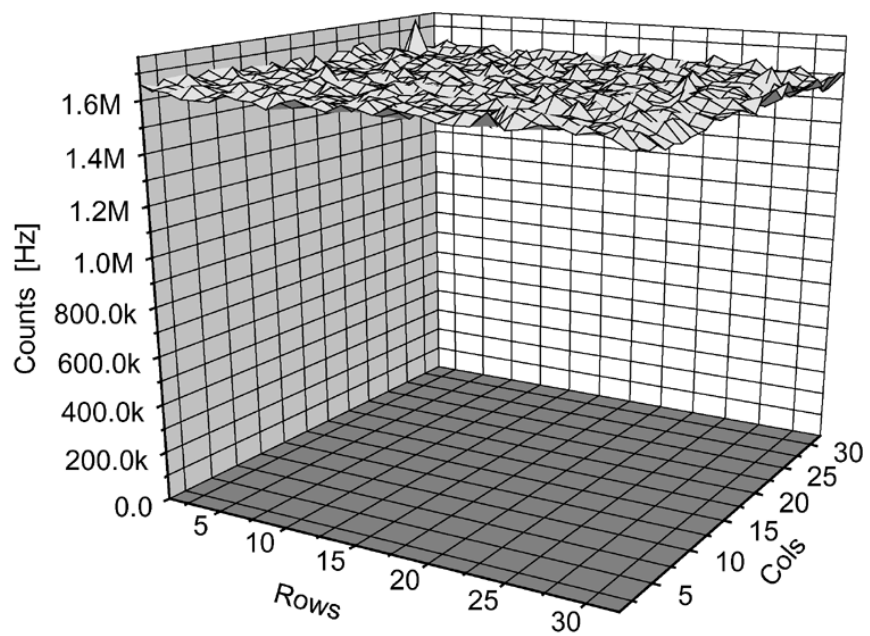

Fig. 8. Photon detection probability uniformity across the sensor.

Fig. 9 plots the distribution of the DCR across the sensor array for the nominal $V_{e}$ at room temperature and at $T=0{ }^{\circ} \mathrm{C}$. At room temperature, the limited active area of the SPAD and the outstanding cleanliness of the CMOS process lead to a mean value of the DCR of $350 \mathrm{~Hz}$ on the whole sensor array and negligible after-pulsing effects. For $T=0^{\circ} \mathrm{C}$, the mean value of the DCR dropped below $75 \mathrm{~Hz}$.

Fig. 10 presents the timing jitter measurement of the pixel. The measurement setup consisted of a pulsed laser source with pulsewidth of $30 \mathrm{ps}$ used to excite a single pixel through an optical fiber. A LeCroy Wavepro $730020 \mathrm{GS} / \mathrm{s}$ oscilloscope was used to measure the time interval between the laser output trigger and the sensor output signal. The resulting overall timing jitter was 115 ps FWHM even though the SPAD jitter is likely to be lower than 50 ps FWHM [11]. The mismatch is likely caused by a non-optimized leading edge rise time in the readout circuitry. More careful design and layout techniques ought to be employed in the readout circuitry if the imager is intended for 3-D vision or time correlated applications. The SPAD jitter is less than half that of the actual image sensor. Thus, a considerable margin for improvement still exists. 

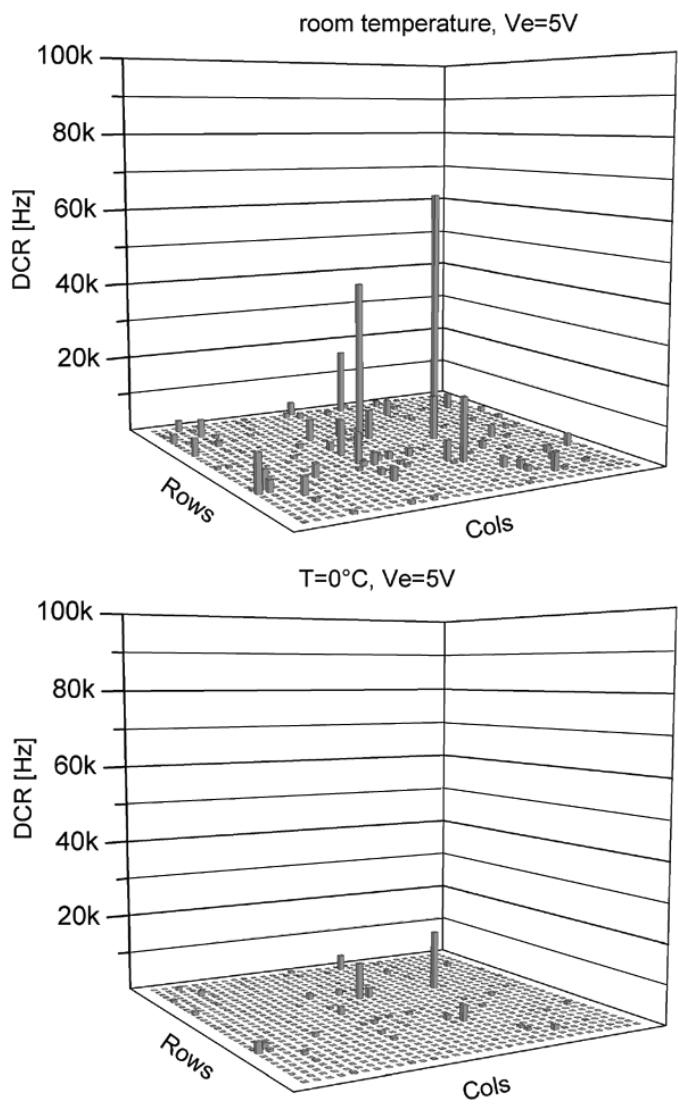

Fig. 9. Dark count rate for nominal excess voltage at room temperature and $T=0{ }^{\circ} \mathrm{C}$

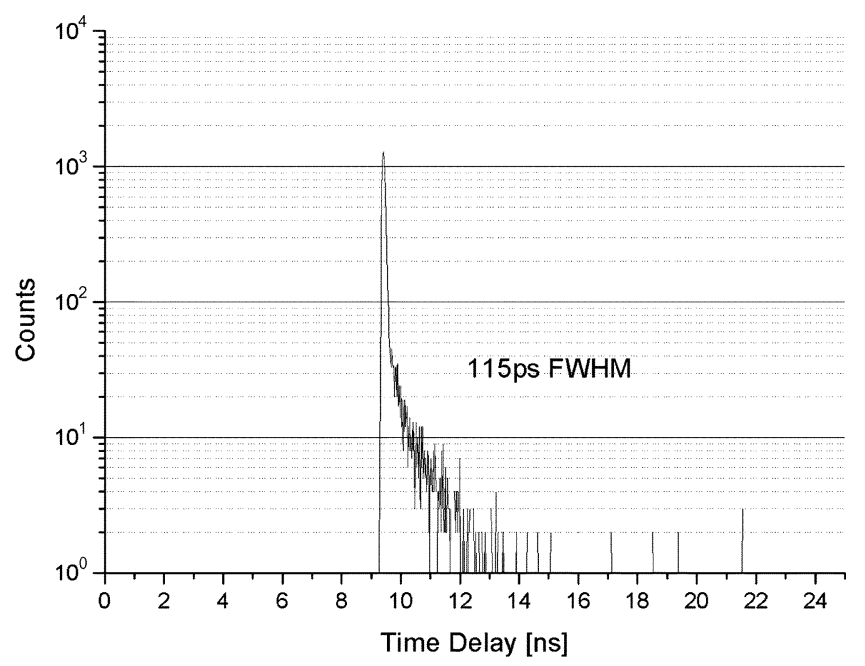

Fig. 10. Histogram of the measured timing jitter (from the slowest row).

Experimental measurements demonstrated that the SPAD array is completely free of crosstalk effects. Crosstalk was measured by illuminating a single pixel in the center of the array using a highly focused laser beam through the optics of a microscope. Fig. 11 shows the resulting image of the crosstalk measurements whereby the excited pixel's neighbors showed a response less than $0.01 \%$ of the center pixel. This value is below DCR levels. As expected, optical crosstalk is negligible since the parasitic capacitance is very small. Electrical crosstalk was

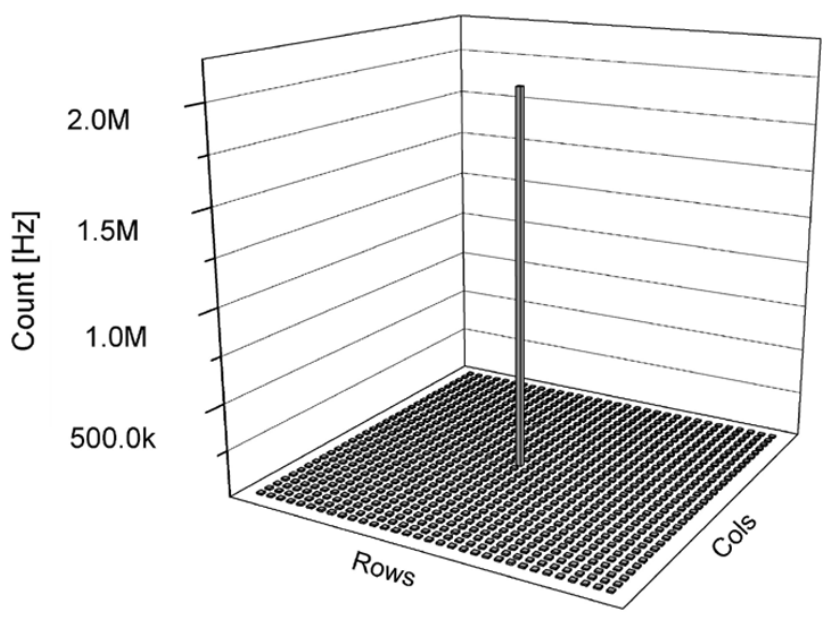

Fig. 11. Crosstalk measurement result.

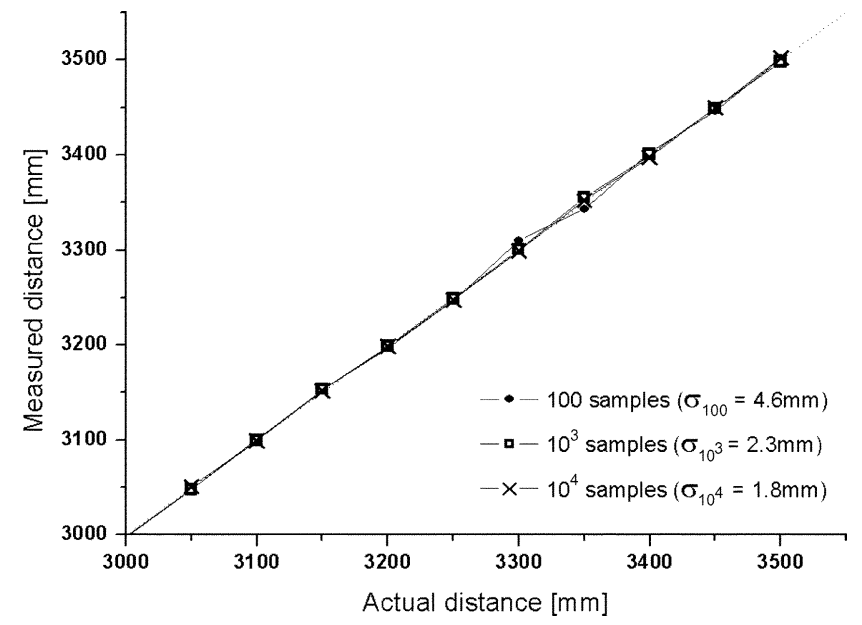

Fig. 12. Distance measurements.

eliminated by design. Minor carriers diffusing in the substrate cannot reach the multiplication region of a pixel since they would be collected by the deep n-tub/p-sub junction.

\section{B. Rangefinder}

Operating in rangefinding mode, a total timing uncertainty $\sigma(\tau)$ of 250 ps was expected whereas 300 ps has been measured. $\sigma(\tau)$ is believed to be dominated by the TDC resolution and the extra jitter produced by digital switching on-chip. Although the measurement was carried out serially, all 1024 SPADs operated simultaneously during the measurement, thus generating noise on substrate and $V_{\mathrm{DD}}$ lines. These noise sources may potentially increase the overall sensor timing jitter. To reach millimetric precision, the proposed processing algorithm was adopted, whereby the threshold value was set to $10 \%$ of the peak's maximum value. Fig. 12 shows multiple depth measurements with $M=100,10^{3}$, and $10^{4}$ at and around 3-m distance. The 1- $\sigma$ uncertainty is also reported.

Next, the depth map of a live-sized human mannequin was captured. Fig. 13 shows a high-resolution picture of the model. To demonstrate the actual $32 \times 32$ pixel resolution, an intensity map of the model, obtained with our sensor operating in 2-D mode, is also shown in the figure. 

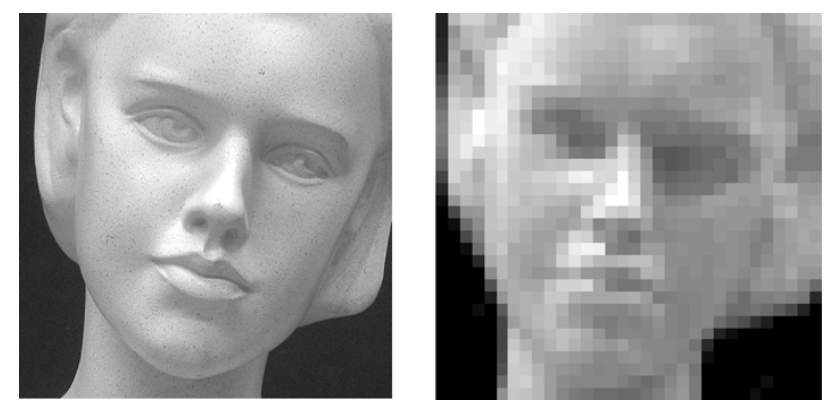

Fig. 13. Model photographed with high-resolution camera (left) and with our camera operating in 2-D mode (right).
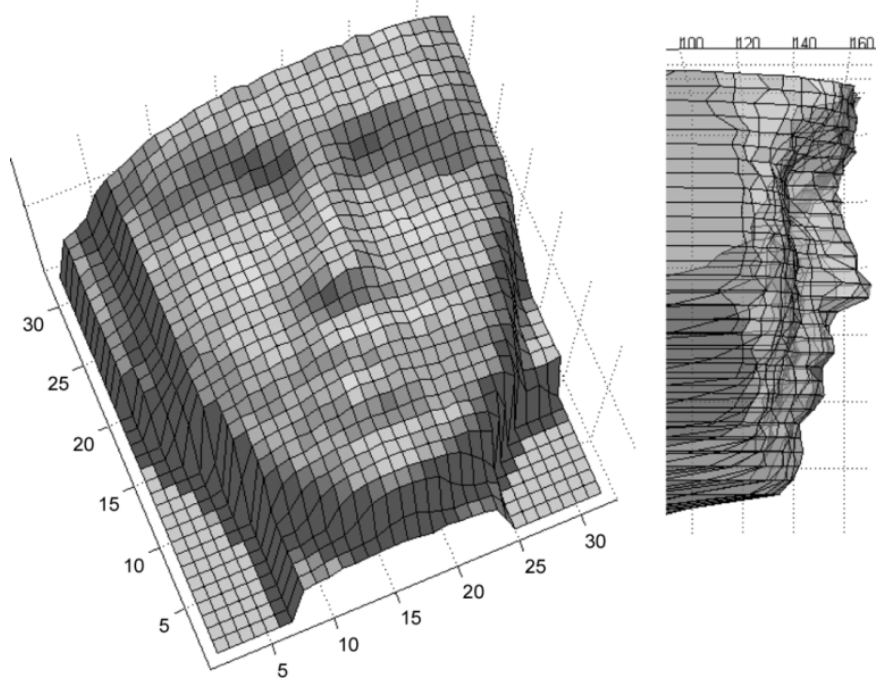

Fig. 14. Human face depth map and profile (in millimeters).

Fig. 14 shows the depth map of the model's face and profile. The model was placed in front of a plane reference at $3 \mathrm{~m}$ from the sensor. The image was obtained using the same light source parameters of the above experiments with $M=10^{4}$. In the current implementation, the average measurement rate was 50000 measurements per second, which gives a pixel acquisition time $t_{\text {acq }}$ of $200 \mathrm{~ms}$. In addition, due to the implemented serial readout circuit, the frame acquisition time was $205 \mathrm{~s}$. Work on a parallel readout circuit is currently under way. This mechanism is expected to considerably increase the sensor frame rate even with larger pixel arrays. Table I lists the salient performance measurements of the image presented in this paper. Note that the mean optical power was only $750 \mu \mathrm{W}$.

\section{CONCLUSION}

Accurate depth mapping of arbitrary 3-D scenes is performed using an imaging system based on time-of-flight. A cone of pulsed light generated by uncollimated laser illuminates the scene. The photons reflected by an object in the scene are collected by the imager that accurately detects their arrival time, thereby inferring the distance to the reflection point. The core of the system is an array of $32 \times 32$ single photon avalanche diodes that allows picosecond-accurate timing measurements at low levels of light intensity, thus achieving millimetric depth resolutions. The range of the depth map is $3 \mathrm{~m}$, while no
TABLE I

PERFORMANCE SUMMARY

\begin{tabular}{|c|c|c|c|c|}
\hline Level & Measure & Symbol & Value & Unit \\
\hline \multirow{5}{*}{ Pixel } & $\begin{array}{l}\text { Photon Detection } \\
\text { probability } @ 635 \mathrm{~nm}\end{array}$ & $\eta$ & 12 & $\%$ \\
\hline & Dark count rate & DCR & 350 & $\mathrm{~Hz}$ \\
\hline & Fill factor & - & 1.1 & $\%$ \\
\hline & FWHM jitter & - & 115 & ps \\
\hline & Dead time & $t_{d}$ & $<40$ & ns \\
\hline \multirow{5}{*}{ Sensor } & Distance range & $D$ & 3 & $\mathbf{m}$ \\
\hline & Time uncertainty & $\sigma(\tau)$ & 300 & ps \\
\hline & RMS Distance accuracy & $\sigma(d)$ & 1.8 & $\mathbf{m m}$ \\
\hline & $\begin{array}{l}\text { Mean number of } \\
\text { measurements per pixel }\end{array}$ & - & $10^{2} \sim 10^{4}$ & - \\
\hline & Power dissipation & $\boldsymbol{P}_{t o t}$ & $<6$ & $\mathbf{m W}$ \\
\hline \multirow{5}{*}{ Source } & Repetition rate & $f_{R}$ & 50 & MHz \\
\hline & Peak power & $\hat{P}_{S}$ & 100 & $\mathrm{~mW}$ \\
\hline & Average power & $P_{S}$ & 750 & $\mu \mathbf{W}$ \\
\hline & Wavelength & $\lambda$ & 635 & $\mathrm{~nm}$ \\
\hline & RMS Pulse width & $T_{p}$ & $<150$ & ps \\
\hline
\end{tabular}

mechanical micro-optical scanning device is required. Due to the high sensitivity of the detectors, very low output optical power is needed by the laser source, thus reducing the overall cost of the system and ensuring strict eye-safe operation.

\section{REFERENCES}

[1] S. Yoshimura, T. Sugiyama, K. Yonemoto, and K. Ueda, "A 48 kframe/s CMOS image sensor for real-time 3-D sensing and motion estimation," in IEEE Int. Solid-State Circuits Conf. (ISSCC) Dig. Tech. Papers, Feb. 2001, pp. 94-95.

[2] J. Dyson, Interferometry as a Measuring Tool. Brighton, U.K.: Machinery Pub., 1970.

[3] R. Lange, " 3D Time-of-Flight Distance Measurement With Custom Solid-State Image Sensors in CMOS/CCD-Technology," Ph.D. dissertation, ETH-Zürich, Switzerland, 2000.

[4] E. Charbon and C. Bamji, " Methods for CMOS-compatible three-dimensional image sensing using quantum efficiency modulation," U.S. Patent 6,515,740, Feb. 4, 2003.

[5] R. Jeremias, W. Brockherde, G. Doemens, B. Hosticka, L. Listl, and P. Mengel, "A CMOS photosensor array for 3D imaging using pulsed laser," in IEEE Int. Solid-State Circuits Conf. (ISSCC) Dig. Tech. Papers, Feb. 2001, pp. 252-253.

[6] B. F. Aull et al., "Geiger-mode avalanche photodiodes for three dimensional imaging," Lincoln Lab. J., vol. 12, no. 2, pp. 335-350, 2002.

[7] J. Massa, G. Buller, A. Walker, G. Smith, S. Cova, M. Umasuthan, and A. M. Wallace, "Optical design and evaluation of a three-dimensional imaging and ranging system based on time-correlated single-photon," Appl. Opt., vol. 41, no. 6, pp. 1063-1070, Feb. 2002.

[8] A. Rochas, "Single Photon Avalanche Diodes in CMOS Technology," Ph.D. dissertation, EPF-Lausanne, Switzerland, 2003.

[9] A. Rochas, M. Gösch, A. Serov, P. A. Besse, R. S. Popovic, T. Lasser, and R. Rigler, "First fully integrated 2-D array of single-photon detectors in standard CMOS technology," IEEE Photon. Technol. Lett., vol. 15, no. 7, pp. 963-965, Jul. 2003.

[10] C. Niclass, A. Rochas, P. A. Besse, and E. Charbon, "A CMOS single photon avalanche diode array for 3D imaging," in IEEE Int. Solid-State Circuits Conf. (ISSCC) Dig. Tech. Papers, Feb. 2004, pp. 120-121.

[11] A. Rochas, M. Gani, B. Furrer, G. Ribordy, P. A. Besse, N. Gisin, and R. S. Popovic, "Single photon detector fabricated in a CMOS high voltage technology," Rev. Sci. Instr., vol. 74, no. 7, pp. 3263-3270, 2003.

[12] R. H. Haitz, "Studies on optical coupling between silicon p-n junctions," Solid State Electron., vol. 8, pp. 417-425, 1965. 
[13] B. Fowler, A. El Gamal, and D. X. D. Yang, "A CMOS area image sensor with pixel-level A/D conversion," in IEEE Int. Solid-State Circuits Conf. (ISSCC) Dig. Tech. Papers, Feb. 1994, pp. 226-227.

[14] D. X. D. Yang, A. El Gamal, B. Fowler, and H. Tian, "A $640 \times 512$ CMOS image sensor with ultrawide dynamic range floating-point pixellevel ADC," IEEE J. Solid-State Circuits, vol. 34, no. 12, pp. 1821-1834, Dec. 1999.

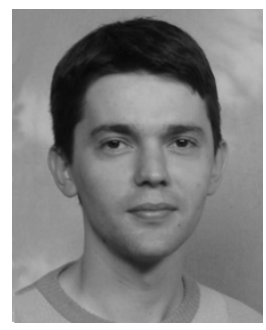

Cristiano Niclass (S’05) received the M.Sc. degree in microtechnology with emphasis in applied photonics from the Swiss Federal Institute of Technology in Lausanne (EPFL) in 2003. During his master degree, he designed a novel electronic payment device which led to the creation of a new company in Switzerland. As a result of its success, the start-up company was eventually acquired months later by Ingenico Group, the worldwide leader in electronic payment. In May 2003, he joined the Processor Architecture Laboratory of EPFL where he is working toward the $\mathrm{Ph} . \mathrm{D}$. degree in electrical and computer engineering.

His interests include the design of high-speed and low-noise digital and mixed-mode application specific integrated circuits. He is currently in charge of the design, implementation, and evaluation of fully integrated two- and three-dimensional CMOS image sensors based on single photon avalanche diodes. Several applications requiring high sensitivity and ultra-fast optical response have been considered.

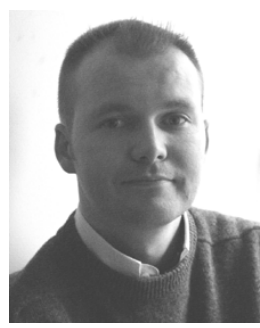

Alexis Rochas was born in Lyon, France, in 1971. In 1995, he received a Master's degree in physics from the University Lyon 1, France. In 1997, he received the Postgraduate Diploma in control sciences and technologies from the Institute of Vision Engineering in Saint-Etienne, France. He received the Ph.D. degree in September 2003.

As a Research Engineer, he worked for Crismatec, a Saint-Gobain subsidiary (France), and was in charge of reducing the production costs of optical crystals. In 1998, he joined the Institute of Microsystems of Swiss Federal Institute of Technology in Lausanne (EPFL) as a Research Assistant. He worked on the development of an UV-sensor microsystem for flame detection in the framework of an industrial collaboration with Siemens Building Technologies, Inc. From June 2000, he worked as a $\mathrm{Ph}$.D. student in the field of single photon avalanche diodes (SPADs) fabricated in standard CMOS technology. He also developed dedicated single photon detectors in collaboration with Siemens Building Technologies for flame detection application, with Gnothis SA, Lausanne, Switzerland, for single molecule detection by fluorescence correlation spectroscopy and with idQuantique, Geneva, Switzerland, for random numbers generation. In November 2003, he joined the Processor Architecture Laboratory of EPFL where he was in charge of designing large array of SPADs in CMOS technology and investigating potential applications. Since July 2004, he has been responsible for the development of single photon detectors at idQuantique. He has published over 30 journal and conference papers.

Dr. Rochas received the 2004 scientific Omega prize for his work in the field of silicon-based single photon detectors.

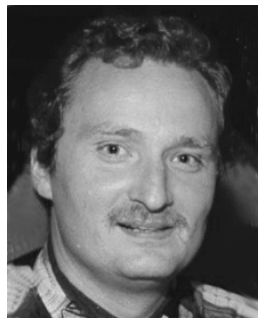

Pierre-André Besse was born in Sion, Switzerland, in 1961. He received the Diploma in physics and the Ph.D. degree in semiconductor optical amplifiers from the Swiss Federal Institute of Technology, ETH Zurich, in 1986 and 1992, respectively.

In 1986, he joined the group of micro- and optoelectronics of the Institute of Quantum Electronics at ETH Zurich, where he is engaged in research in optical telecommunication science. He worked on theory, modeling, characterization and fabrication of compound semi-conductor devices. In August 1994, he joined the Institute of Microsystems at the Swiss Federal Institute of Technology in Lausanne (EPFL) as a Senior Assistant, where he starts activities on sensors and actuators microsystems. His major fields of interest are physical principles and new phenomena for optical, magnetic, and inductive sensors. He has authored or co-authored over 100 scientific papers and conference contributions.

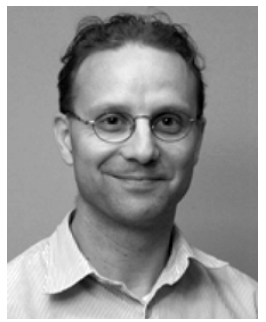

Edoardo Charbon (M'92) received the Diploma in electrical engineering from the Swiss Federal Institute of Technology (ETH), Zurich, in 1988, the M.S. degree in electrical and computer engineering from the University of California at San Diego in 1991, and the Ph.D. degree from the University of California at Berkeley in 1995. His doctoral work focused on performance-directed constraint-based analog and mixed-signal physical design automation and accelerated substrate extraction techniques.

From 1995 to 2000, he was with Cadence Design Systems, where he was the architect of the company's initiative for intellectual property protection. In 2000, he joined Canesta Inc. as its Chief Architect, leading the development of wireless three-dimensional CMOS image sensors. Since November 2002, he has been a member of the Faculty of the Swiss Federal Institute of Technology, working in the field of 3-D sensors and ultra low-power wireless embedded systems. He has consulted for numerous organizations, including Texas Instruments Incorporated, Hewlett-Packard, and the Carlyle Group. He has published over 60 articles in technical journals and conference proceedings and two books, and he holds six patents. His research interests include 3-D micro-imaging, radio-frequency integrated circuits, intellectual property protection, substrate modeling and characterization, and micromachined sensor design.

Dr. Charbon has served as Guest Editor of the IEEE TRANSACTIONS ON COMPUTER-AIDED DESIGN OF INTEGRATED CIRCUITS AND SYSTEMS and as a Member of the Technical Committee of the IEEE Custom Integrated Circuits Conference since 1999. 